\title{
Article \\ Spine, Pelvis and Hip Kinematics-Characterizing the Axial Plane in Healthy and Osteoarthritic Hips
}

\author{
Danilo S. Catelli ${ }^{1}$ D, Brendan Cotter ${ }^{2}$, Mario Lamontagne ${ }^{1,2, *(\mathbb{D})}$ and George Grammatopoulos ${ }^{2, *(D)}$ \\ 1 Human Movement Biomechanics Laboratory, Faculty of Health Sciences, School of Human Kinetics, \\ University of Ottawa, Ottawa, ON K1N 6N5, Canada; danilo.catelli@uottawa.ca \\ 2 Division of Orthopaedic Surgery, University of Ottawa, Ottawa, ON K1N 6N5, Canada; bcotter@ohri.ca \\ * Correspondence: mlamon@uottawa.ca (M.L.); ggrammatopoulos@toh.ca (G.G.)
}

Citation: Catelli, D.S.; Cotter, B.; Lamontagne, M.; Grammatopoulos, G. Spine, Pelvis and Hip KinematicsCharacterizing the Axial Plane in Healthy and Osteoarthritic Hips. Appl. Sci. 2021, 11, 9921. https:// doi.org/10.3390/app11219921

Academic Editors: Alberto Leardini and Zimi Sawacha

Received: 30 September 2021

Accepted: 21 October 2021

Published: 23 October 2021

Publisher's Note: MDPI stays neutral with regard to jurisdictional claims in published maps and institutional affiliations.

Copyright: (c) 2021 by the authors. Licensee MDPI, Basel, Switzerland. This article is an open access article distributed under the terms and conditions of the Creative Commons Attribution (CC BY) license (https:// creativecommons.org/licenses/by/ $4.0 /)$.
Abstract: Abnormal spinopelvic movements are associated with inferior outcomes following total hip arthroplasty (THA). This study aims to (1) characterize the agreement between dynamic motion and radiographic sagittal assessments of the spine, pelvis, and hip; (2) determine the effect of hip osteoarthritis (OA) on kinematics by comparing healthy individuals with pre-THA patients suffering from uni- or bilateral hip OA. Twenty-four OA patients pre-THA and eight healthy controls underwent lateral spinopelvic radiographs in standing and seated bend-and-reach (SBR) positions Lumbar-lordosis (LL), sacral-slope (SS), and pelvic-femoral (PFA) angles were measured in both positions, and the differences $(\Delta)$ between SBR and standing were computed to assess spine flexion (SF), pelvic tilt (PT), and hip flexion (HF), respectively. Dynamic SBR and seated maximal trunk rotation (STR) tasks were performed at the biomechanics laboratory. Peak sagittal and axial kinematics for spine, pelvis, and hip, and range of motion (ROM), were calculated for SBR and STR. Radiograph readings correlated with sagittal kinematics during SBR for $\Delta \mathrm{LL}$ and SFmax $(r=0.66, p<0.001)$, $\triangle \mathrm{PT}$ and PTmax $(r=0.44, p=0.014)$, and $\triangle \mathrm{PFA}$ and HFmax $(r=0.70, p<0.001)$, with a satisfactory agreement in Bland-Altman analyses. Sagittal SBR spinal $(r=0.33, p=0.022)$ and pelvic $(r=0.35$, $p=0.018)$ flexions correlated with the axial STR rotations. All axial spinopelvic parameters were different between the OA patients and controls, with the latter exhibiting significantly greater mobility and less variability. Bilaterally affected patients exhibited lower peak and ROM compared to controls. The biomechanics laboratory performed reliable assessments of spinopelvic and hip characteristics, in which the axial plane can be included. The sagittal and axial pelvic kinematics correlate, illustrating that pelvic rotation abnormalities are likely also contributing to the inferior outcomes seen in patients with abnormal spinopelvic flexion characteristics. Axial rotations of the pelvis and spine were least in patients with bilateral hip disease, further emphasizing the importance of the hip-pelvic-spine interaction.

Keywords: hip osteoarthritis; kinematics; axial plane; motion analysis; spinopelvic mobility

\section{Introduction}

The dynamic interaction between the femur, pelvis, and lumbar spine has been an emerging issue amongst hip and spine surgeons. The importance of this interaction was first highlighted by observational studies that reported inferior outcomes following hip arthroplasty in patients with spinal arthrodesis. Patients with spinal arthrodesis exhibit abnormal spinopelvic movement and have been shown to be at increased risk of dislocation following total hip replacement (THA) [1,2]. Furthermore, observational radiographic studies have illustrated a wide variability in spinopelvic characteristics among THA patients [3].

These observations [4,5] emphasize the relevance of considering the spinopelvic characteristics in all arthroplasty patients, as they determine the position of the pelvis in space, which, in turn, determines the functional acetabular orientation. Acetabular 
component orientation is an important determinant of hip function and has been shown to influence risk of wear $[6,7]$, instability $[6,8,9]$, impingement [6], and patient-reported outcome measures [8]. Modelling studies have illustrated that the motion of the pelvis along each plane has a varying effect on acetabular component orientation [10]. Pelvic obliquity (motion about the frontal (coronal) plane) primarily affects cup inclination; pelvic tilt (motion along the sagittal plane) primarily alters version; and pelvic rotation (motion along the transverse plane) changes both version (mostly) and inclination [11].

To date, all assessments have been performed in the frontal and sagittal planes as these two planes can be adequately studied with anteroposterior and lateral radiographs, respectively $[4,6,12,13]$. However, very little information exists on what movements take place in the axial plane and how these may relate to the ones measured in the sagittal plane. Abnormal rotation movements of the pelvis about the transverse plane are likely to influence the outcome, as they have a significant effect on cup orientation and thus determine impingement risk. The study of axial plane movements during activities of daily living is not possible with radiographs. Thus, if these movements are to be best studied dynamically, a motion capture analysis may be the most appropriate setting. However, this has not been tested to date in arthroplasty patients. This prospective study aims to (1) examine the agreement between axial movement measured from the motion capture and the range of motion measured from sagittal radiographs of the spine, pelvis, and hip; and (2) determine the effect of hip osteoarthritis $(\mathrm{OA})$ on the spine, pelvis, and hip kinematics by comparing asymptomatic volunteers with pre-THA patients suffering from unilateral or bilateral hip osteoarthritis (OA).

\section{Materials and Methods}

\subsection{Study Design}

This is a prospective, diagnostic, IRB-approved cohort study from an academic, tertiary referral center. The study was conducted according to the Helsinki Declaration with all participants providing informed consent. The study included patients with hip OA due to undergo THA and a group of healthy volunteers without hip or spinal pathology. The participants were invited to take part in a radiographic and a three-dimensional motion capture session. Exclusion criteria included age above 85 years old, history of dementia, previous history of spinal pathology or surgery, a body mass index (BMI) above 35 (precludes visibility of markers at motion analysis laboratory), and an inability to perform tasks of daily living without the use of aids.

\subsection{Patients}

All patients were recruited from arthroplasty clinics of the tertiary hospital. Twentyfour patients with symptomatic hip OA due for a THA agreed to participate. Seven of the patients suffered from bilateral OA, and the remaining 17 suffered from unilateral OA.

\subsection{Controls}

The healthy volunteers were health care workers recruited from the arthroplasty clinic and the operating room of the local hospital. A total of 10 volunteers were asymptomatic with regard to their hips (Oxford hip score of greater than 45/48) and spine (Oswestry disability index less than 5\%). However, to improve age matching between cohorts, 2 volunteers below the age of 40 years old were excluded from the analysis.

\subsection{Clinical Review}

All participants were asked to complete four validated patient reported outcome measure (PROMs) questionnaires assessing hip and spine function as well as overall wellbeing, including the Oxford hip score [14], the HOOS score [15], the Oswestry disability index [16], and the SF-12 physical score [17]. Detailed demographics and PROMs are provided in Tables 1 and 2, respectively. 
Table 1. Summary of participants' demographics, reporting mean (range).

\begin{tabular}{cccc}
\hline Parameters & OA & Control & $p$-Value \\
\hline Participants $(\mathrm{n})$ & 24 & 8 & - \\
Sex (F/M) & $12 / 12$ & $4 / 4$ & - \\
Age (years) & $67(48-84)$ & $58(40-66)$ & $\mathbf{0 . 0 3}$ \\
Height $(\mathrm{m})$ & $1.68(1.54-1.85)$ & $1.69(1.56-1.88)$ & 0.79 \\
Weight $(\mathrm{kg})$ & $74(47-97)$ & $70(54-87)$ & 0.59 \\
BMI $\left(\mathrm{kg} / \mathrm{m}^{2}\right)$ & $26(18-33)$ & $24(20-29)$ & 0.23 \\
\hline
\end{tabular}

Table 2. Summary of participants' patient-reported outcome measures, reporting mean (range).

\begin{tabular}{|c|c|c|c|c|}
\hline & Parameters & OA & Control & $p$-Value \\
\hline \multirow{5}{*}{ HOOS } & Symptoms & $39(5-55)$ & $99(90-100)$ & $<0.001$ \\
\hline & Pain & $40(13-63)$ & $98(88-100)$ & $<0.001$ \\
\hline & Activities of Daily Living & $44(16-69)$ & $100(99-100)$ & $<0.001$ \\
\hline & Sports and Recreational Activities & $22(0-50)$ & $100(100-100)$ & $<0.001$ \\
\hline & Quality of Life & $21(0-50)$ & $97(75-100)$ & $<0.001$ \\
\hline \multicolumn{2}{|r|}{ SF-12 physical } & $34(28-40)$ & $42(39-47)$ & $<0.001$ \\
\hline \multicolumn{2}{|r|}{ Oswestry spinal score } & $34(4-68)$ & $0(0-2)$ & $<0.001$ \\
\hline \multicolumn{2}{|r|}{ Oxford hip score } & $21(9-36)$ & $48(46-48)$ & $<0.001$ \\
\hline
\end{tabular}

\subsection{Radiographic Assessments}

All participants underwent two lateral spinopelvic radiographs of pelvis, hip, and proximal femur in (1) standing spine; and (2) seated deep flexed forward. This was chosen because it is considered a position of increased dislocation risk and has been shown to better characterize the spinopelvic characteristics of THA patients and assess maximum lumbar flexion [18,19]. Radiographic measurements of the sacral slope (SS), pelvic-femoral angle (PFA) and lumbar lordosis (LL) angles were performed in both positions, as defined in Figure 1. All assessments were measured by a single observer twice. The changes in SS equal the changes in pelvic tilt (PT).

The differences in sagittal angles (LL, SS, and PFA) measured between the two positions were calculated [2] as follows:

$$
\begin{aligned}
& \text { Sagittal radiographic lumbar flexion }=\Delta L L=L L_{\text {standing }}-L_{\text {seated }} \\
& \text { Sagittal radiographic pelvic tilt }=\Delta \mathrm{PT}=\Delta S S=S_{\text {standing }}-S_{\text {seated }} \\
& \text { Sagittal radiographic hip flexion }=\Delta \mathrm{PFA}=\mathrm{PFA}_{\text {seated }}-\mathrm{PFA}_{\text {standing }}
\end{aligned}
$$

\subsection{Motion Analysis}

After the radiographic assessment, all volunteers underwent motion analysis testing in a biomechanics laboratory. They were instructed to warm up for five minutes on a cycle ergometer and then outfitted with 45 reflective markers according to the University of Ottawa motion analysis model (UOMAM) marker set [20]. The participants were initially asked to take a neutral, standing static pose, and then they dynamically performed three trials of seated bend and reach (SBR) and seated maximal trunk rotation (STR) as shown in Figure 2. 


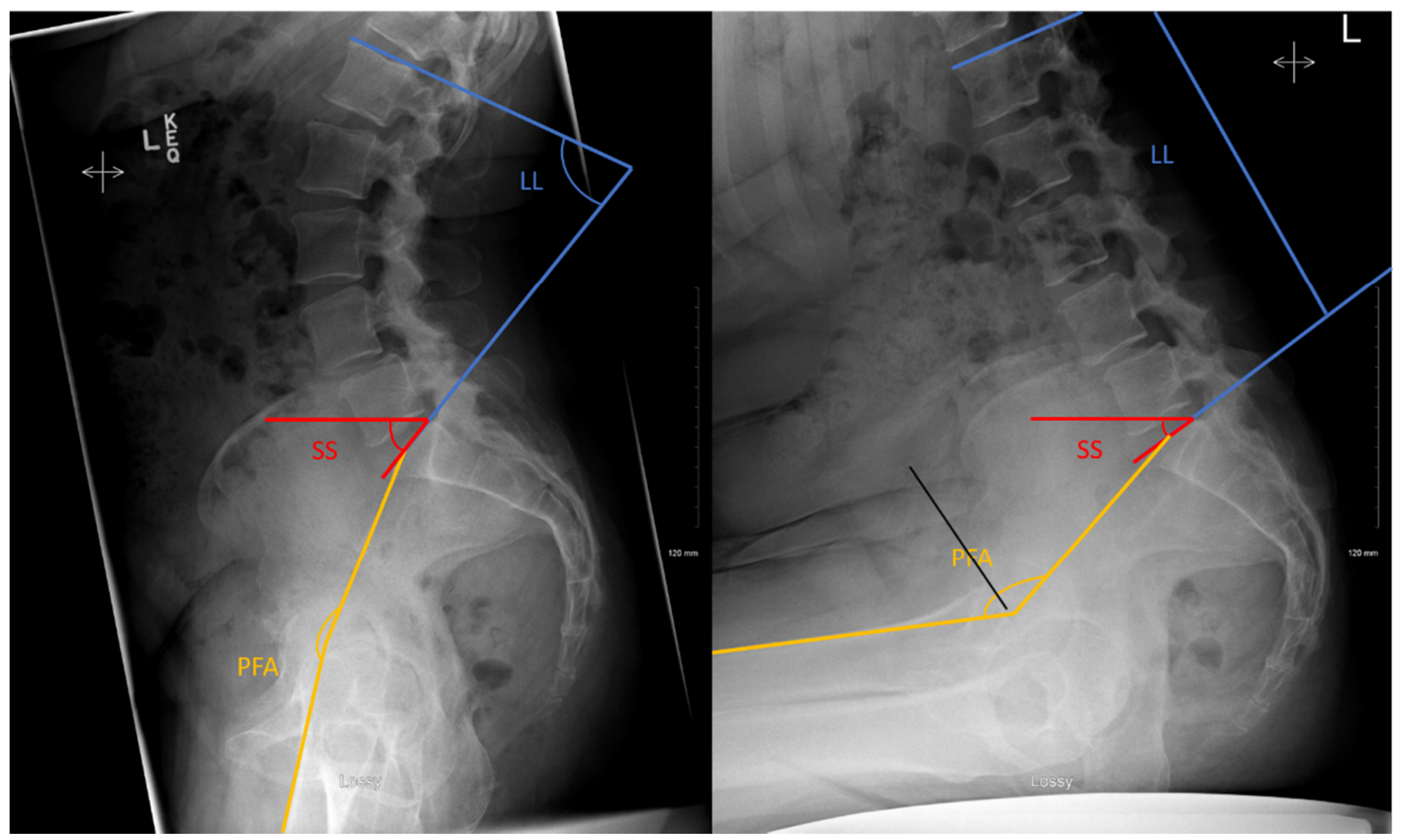

Figure 1. Lateral view of the lumbar spine and pelvis from the standing (left) and seated deep flexed forward (right). With angles calculated for sacral slope (SS), which is the angle tangent to the S1 endplate and a transverse line; pelvic femoral angle (PFA) defined as an angle based on a line from the center of the S1 endplate to the center of the femoral head and a second line that parallels the diaphysis of the femur; lumbar lordosis (LL) being a line tangent to the S1 endplate relative to a line tangent to the superior L1 endplate. The changes in SS were considered equivalent to the changes in pelvic tilt (PT).
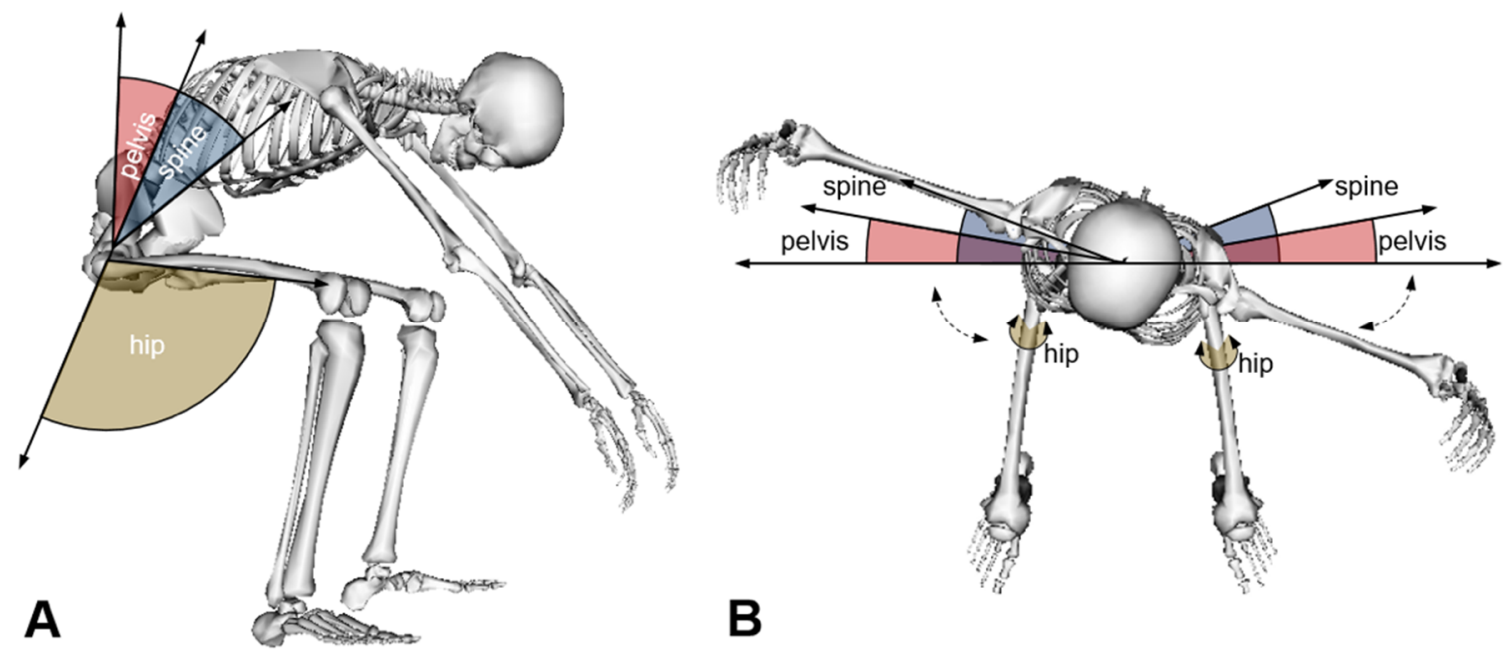

Figure 2. (A) Lateral view representation of sagittal joint kinematics assessment during SBR: spine flexion, pelvis tilt, and hip flexion. Spine flexion is the angle between the sagittal thorax axis and the sagittal pelvis axis around the fixed transverse axis of the pelvis. Pelvic tilt is measured as the angle in the transverse plane between the projected sagittal pelvic axis and the sagittal laboratory axis. Hip flexion is the angle between the projected sagittal thigh axis and the sagittal pelvic axis. (B) Superior view representation of axial joint kinematics assessment during STR: left and right spine rotations, left and right pelvis rotations, and left and right hip rotations. Spine rotation is measured as the angle between the sagittal axis of the thorax and the sagittal axis of the pelvis, around a floating frontal axis. Pelvis rotation is the angle measured between the sagittal axis of the pelvis and the sagittal laboratory axis projected into the pelvis transverse plane. Hip rotation is calculated between the sagittal axis of the thigh and the sagittal axis of the pelvis projected into the plane perpendicular to the long axis of the thigh. 


\section{Seated Bend and Reach (SBR)}

The seat height was adjusted according to participants' knee plateau height. Participants initiated the task seated with their hands placed laterally and were instructed to bend their trunk forward and try to touch the ground as far as comfort allowed, as if they were about to tie their shoelaces. They would have to remain in that position for $5 \mathrm{~s}$ and would thereafter extend their trunk back to the initial seated position, with their hands placed laterally. Participants were instructed to keep their buttocks on the bench during the entire trial. Sagittal movements were captured from the seated upright to the maximum bend and reach position (SBR) and back to the seated upright position-Figure 2A.

\section{Seated Maximal Trunk Rotation (STR)}

Participants remained seated on the adjusted knee height bench in the upright position, with their elbows flexed at 90 degrees and fingers pointing to the ceiling. They were then asked to rotate to the left and to the right as far as possible to determine their maximum range of motion (ROM) in the axial plane. They were instructed to keep their buttocks in contact with the bench. Axial movements were captured from the seated upright to the maximal trunk rotation to the left, followed by the maximal rotation to the right, and back to the seated upright position-Figure 2B.

A ten-camera infrared motion analysis system (two Vantage V5 and eight Vero v2.2, Vicon, UK) was used to track the 45 markers at a sample rate of $200 \mathrm{~Hz}$. The markers were labelled and reconstructed, and the 3D marker trajectories were filtered using a Woltring filtering routine set at a mean square error of $15 \mathrm{~mm}^{2}$. The 3D trajectories were processed by the UOMAM model to provide spatial spine, pelvis, and hip joint kinematics. The joint kinematics were exported to a custom script (Matlab R2019b, Matworks Inc., Beltsville, MD, USA) to extract sagittal and axial kinematics for the spine (mid-thoracic to L-sacral junction), pelvis, and hip.

Parameters of interest for further analysis included the sagittal joint angles of the spine, pelvis, and hip for the SBR task and axial spine and pelvis angles during the STR task. In the sagittal plane, we determined the maximal pelvic tilt (PTmax), hip flexion (HFmax), and spinal flexion (SFmax) reached when the participants were fully bent forward during the SBR task, which was determined relative to the standing static pose. In the coronal plane, during STR task, we determined the maximal left and right rotations for the spine and pelvis, as well as the total axial range of motion (ROM) and the sum of maximal left and right-Figure 3. Still during the STR, the mean of the affected hip rotation was assessed instead for group comparison.

\subsection{Data Analysis}

The maximal pelvic tilt (PTmax), maximal hip flexion (HFmax), and maximal spinal flexion (SFmax) during SBR motion analysis capture laboratory tasks were tested for correlation using Spearman's $r$ with the respective radiographic measurements $(\triangle \mathrm{PT}$, $\Delta \mathrm{PFA}, \Delta \mathrm{LL}$ ). Bland-Altman scatter plots [21] were used to calculate the limits of agreement between the two methods. Spearman- $\rho$ correlations were used to determine relations between the range of motion (ROM) of the spine in sagittal plane of the SBR and in axial plane of the STR. Axial and sagittal, and pelvic and spinal ROM were compared between OA and controls (2-group analysis) and between controls, unilateral, and bilateral osteoarthritic hips (3-group analysis). Mann-Whitney $U$ tests were used to compare kinematic measures between OA and control groups, and Kruskal-Wallis tests were used for 3-group analyses. Significance was set at $p<0.05$. All analyses were performed using IBM SPSS (IBM Corp, Armonk, NY, USA) software for Mac 9 (version 27). 


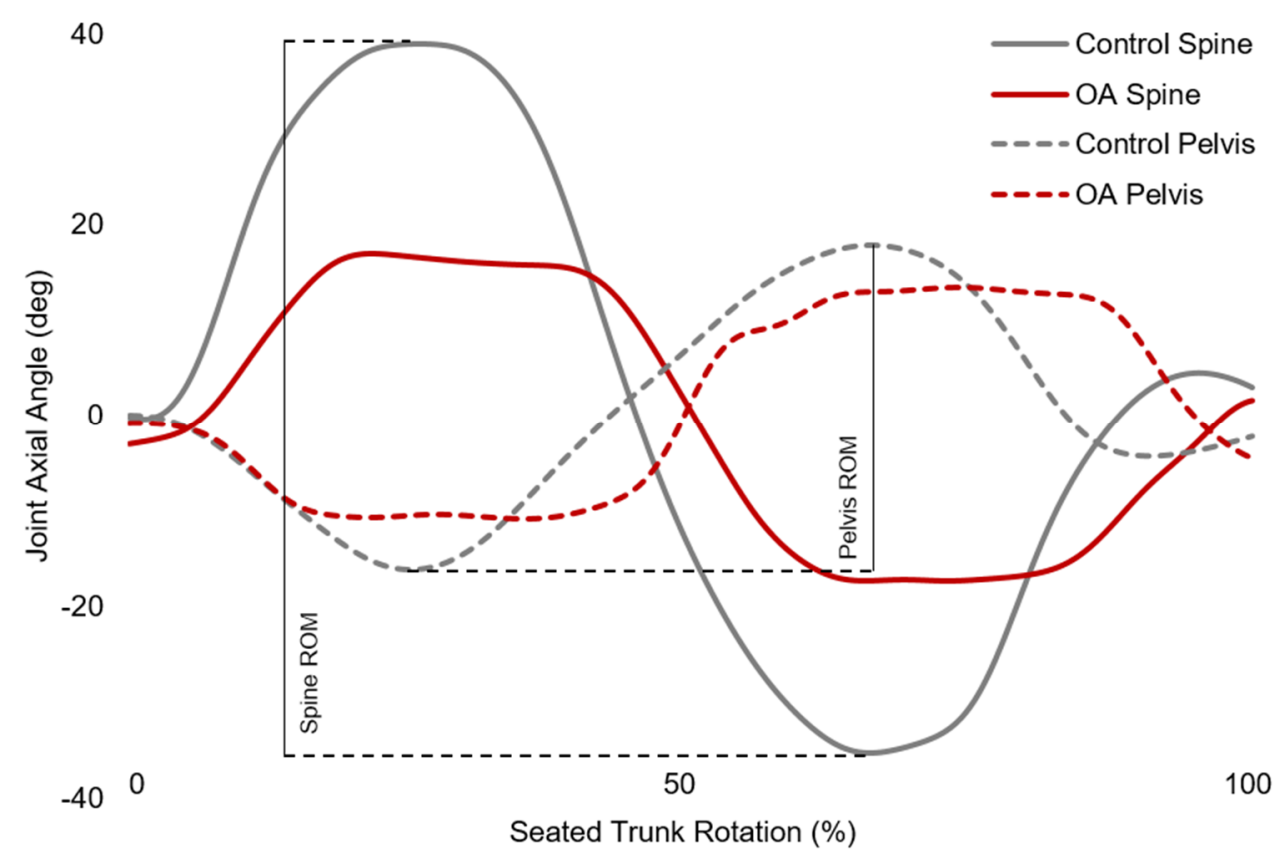

Figure 3. Kinematic axial spine (solid lines) and pelvis (dashed lines) assessments during a time-normalized (0-100\% cycle) Seated Trunk Rotation (STR) task, as an example of data assessment for one control and one osteoarthritis (OA) participants. The thin vertical black lines demonstrate how range of motion (ROM) were assessed.

\section{Results}

\subsection{Clinical Outcomes}

Significant differences in the PROMS were seen between the OA patients and the healthy volunteers (Table 2).

\subsection{Internal Validity of Motion Analysis Measurements}

Radiographic and motion analysis measurements of spine-, pelvis-, and hip- flexion are included in Table 3. Significant and strong correlations were identified between radiographic and motion capture analysis for maximum spinal flexion $(r=0.66, p<0.001)$ and maximal hip flexion $(r=0.70, p<0.001)$. The correlation for maximum pelvic tilt between radiographic and motion analysis measurement was significant but moderate $(r=0.44$, $p=0.014)$. Bland-Altman analyses showed satisfactory interval of agreement for the spine flexion, pelvic tilt, and hip flexion compared to the $\triangle \mathrm{LL}, \triangle \mathrm{PT}$, and $\triangle \mathrm{PFA}$ measured in quasi-static radiography readings-Figure 4.

Table 3. Summary of participants' radiography and motion capture analysis assessments, correlation coefficients $(r)$, and correlation significance, reporting mean (range).

\begin{tabular}{ccccc}
\hline Parameters & OA (deg) & Control (deg) & rho & $p$-Value \\
\hline $\mathrm{SF}_{\max }$ & $53.1(28-70)$ & $55.7(40-74)$ & 0.66 & $<\mathbf{0 . 0 0 1}$ \\
$\Delta \mathrm{LL}$ & $49.8(22-77)$ & $58.4(45-67)$ & & \multirow{2}{*}{0.014} \\
\hline $\mathrm{PT}_{\max }$ & $4.7(-27-27)$ & $15.2(3-29)$ & & \\
$\Delta \mathrm{PT}$ & $3.2(-28-24)$ & $19.4(11-32)$ & 0.44 & $<\mathbf{0 0 1}$ \\
\hline $\mathrm{HF}_{\max }$ & $78.3(46-111)$ & $99.2(83-119)$ & & \\
$\Delta \mathrm{PFA}$ & $83.5(59-102)$ & $100.9(83-113)$ & &
\end{tabular}



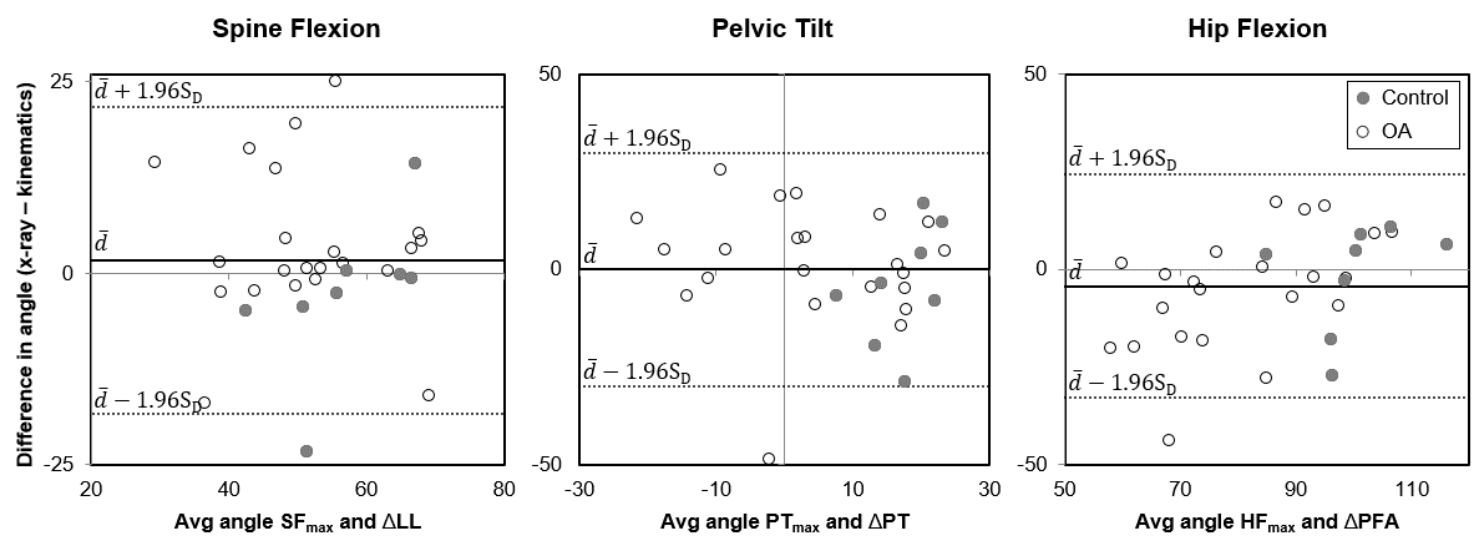

Figure 4. Bland-Altman scatter plots for the joint angle assessments in the radiographic measurements (i.e., $\Delta \mathrm{LL}, \Delta \mathrm{PT}$, and $\triangle \mathrm{PFA}$ ) versus the motion capture system (i.e., SFmax, PTmax, and HFmax) for the three joints of interest: spine, pelvis, and hip. The solid line is the zero-error line $(\bar{d})$; the dotted lines are the limits of agreements $(\bar{d} \pm 1.96 \mathrm{SD})$. Grey markers $=$ control participants; white markers = OA patients.

\subsection{Correlation between Sagittal (SBR) and Axial (STR) Kinematics}

Sagittal spinal flexion ROM during SBR showed moderate and significant correlation with the axial spine rotation ROM during STR $(r=0.33, p=0.022)$. Moderate correlation, and still significant, was also observed between pelvic tilt and pelvic rotation during SBR and STR, respectively $(r=0.35, p=0.018)$.

\subsection{Sagittal and Axial Kinematics in $O A$ and Controls}

All kinematics means and ranges for SBR and STR tasks are reported in Appendix A Table A1.

\subsubsection{Sagittal Plane}

During the SBR activity, patients with hip OA demonstrated significantly reduced hip flexion (78.3 $\pm 19.8 \mathrm{deg})$, compared to controls $(99.2 \pm 13.1 \mathrm{deg}, p=0.01)$. Sagittal spinal $(p=0.782)$ and pelvic $(p=0.113)$ ROM measurements were no different between arthritic patients and controls when transitioning from the seated to the bend-and-reach position (Figure 5A).
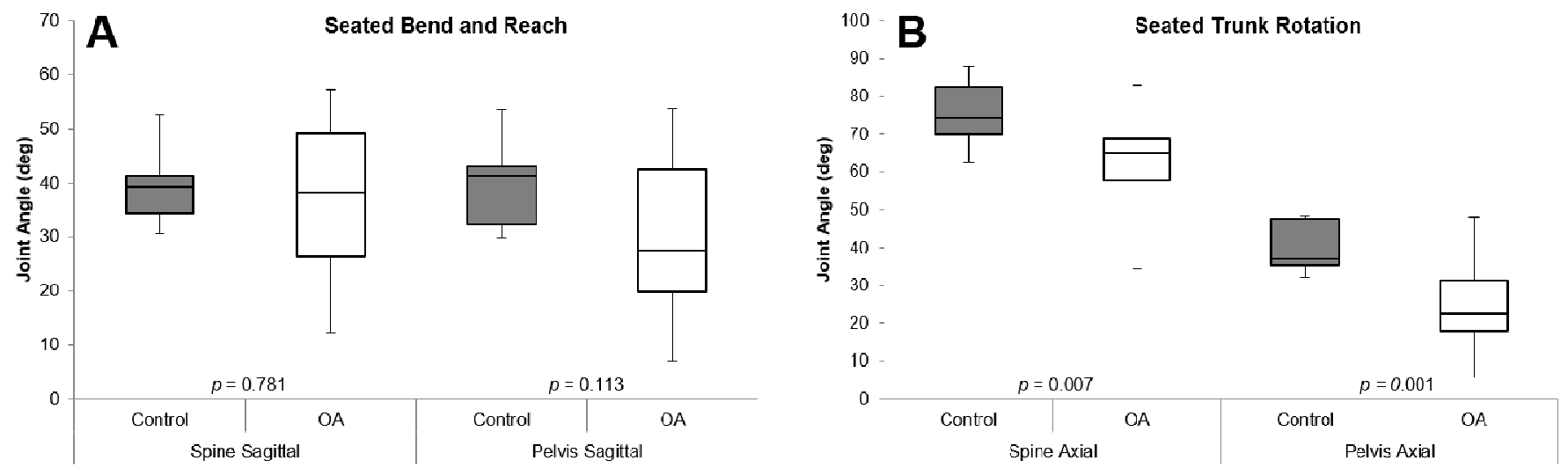

Figure 5. (A) Seated bend and reach (SBR) task range of motion (ROM) of the sagittal spine and pelvis assessments; (B) seated trunk rotation (STR) task axial ROM of the spine and pelvis for the control (dark grey) and osteoarthritis (OA) (white) participants. 


\subsubsection{Axial Plane}

During STR, the OA patients showed significantly reduced axial ROM for both spine $(p=0.007)$ and pelvis $(p=0.001)$ compared to the controls-Figure 5B. In unilaterally arthritic patients, reduced axial kinematics of the spine and pelvis were seen when rotating towards or away from the symptomatic side (Table A1). There was no difference in the rested axial position of the hip when seated between the groups (OA: $3 \pm 10 \mathrm{deg}$, controls: $2 \pm 15 \mathrm{deg} ; p=0.67)$.

Patients with bilateral hip OA exhibited the least maximal and ROM axial rotation of the spine and the pelvis compared to patients with unilateral hip OA and controls (Figure 6).

Seated Trunk Rotation
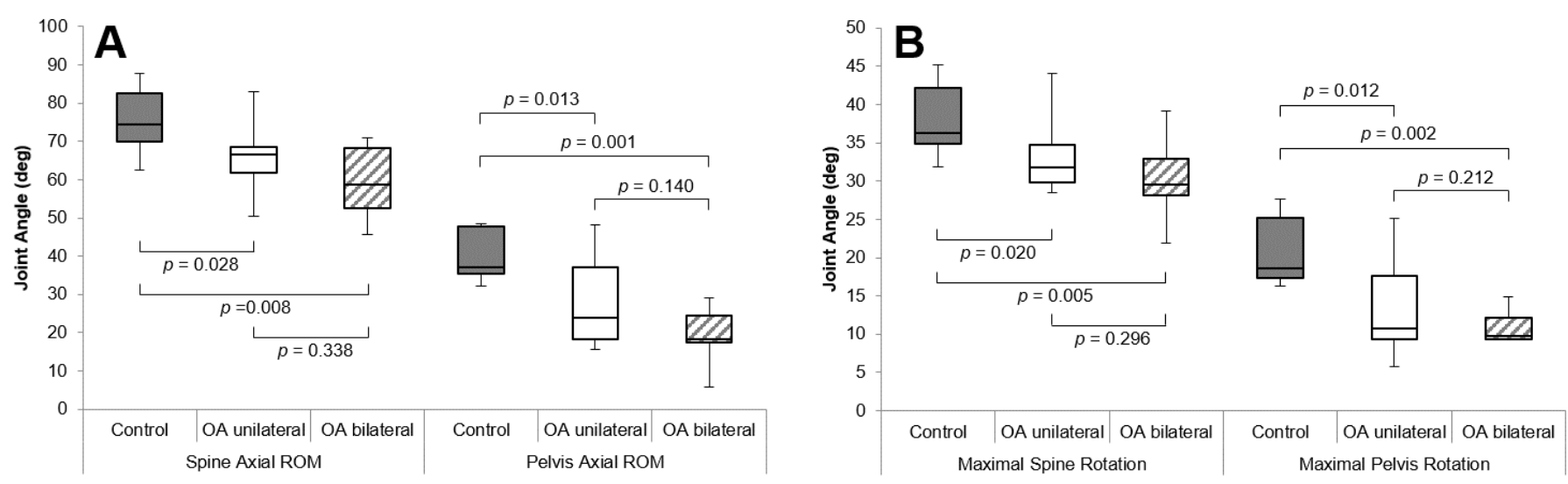

Figure 6. (A) Range of motion (ROM) of the axial spine and pelvis assessments during the seated trunk rotation (STR) task; (B) maximal axial spine and pelvis rotation assessments during the seated trunk rotation (STR) task, for the control (dark grey), unilaterally affected osteoarthritis (OA) (white), and bilateral OA (dashed) participants.

\section{Discussion}

Spinopelvic assessments have been suggested to be included in the pre-operative algorithms by various authors $[4,5,19]$. However, assessments to date have been of the sagittal plane, and no information exists as to what happens in the axial/transverse plane, despite the appreciation of pelvic rotation having an equal effect on cup anteversion to pelvic tilt, thus influencing impingement and dislocation risk [22]. Given that axial assessments cannot be performed radiographically, we sought alternative methods of characterizing the range of movement taking place in this plane and tested the agreement between the measurements performed. Our findings show that assessments of spinopelvic characteristics in the motion analysis laboratory can produce reliable results, albeit with certain inherent limitations pertinent to the assessment of the pelvic, sagittal kinematics.

The motion analysis laboratory has previously been used to study sagittal pelvic and hip range of motions [23]. The agreements illustrated between sagittal plane kinematics and radiographical measurements whilst performing the SBR task validates the ability to perform spinopelvic mobility and hip flexion assessments in the motion analysis laboratory. In particular, hip and spinal flexions showed strong correlations between motion laboratory and radiographic analyses. The pelvic maximum flexion motion illustrated the least agreement. This could be explained by the soft tissue movement artifacts due to the increased soft tissue envelope around the pelvis [24]. This occurred despite the creation of virtual markers associated with the pelvis segment to recreate the position of the missing markers minimize the effect of the soft tissue artifact and selecting participants with $\mathrm{BMI}<35$. The accurate assessment of the pelvis during a bend and reach assessment has certain inherent limitations that are difficult to overcome in the motion laboratory unless using dual fluoroscopy. 
The weak correlation between axial and sagittal spinal kinematics is, in part, due to the type of assessments performed. Spinal flexion mostly occurs in the lumbar region, whilst spinal rotation is minimal in the lumbar spine and mostly occurs in the thoracic region. We thus feel that the weak correlation is primarily due to different segments of the spine responsible for the motion. Thus, patients who illustrate spinopelvic abnormalities in the sagittal plane, when assessed radiographically, are also likely to be illustrating abnormal movements in the axial plane. This is unsurprising, as the abnormal spinopelvic characteristics, particularly spinopelvic hypermobility, in pre-THA patients are, in part, due to the arthritic hip and reflect compensatory maneuvers of the pelvis to accommodate for the reduced range of movement the hip can perform [13]. Further study is required to characterize spine, pelvis, and hip ROM in the axial and sagittal plane during gait and for activities that require rotation in standing position. The STR task keeps the hip relatively static, as the subject is sitting, and thus the extent of rotation can be thoroughly tested.

This study wanted to characterize the sagittal and axial plane kinematics in a spectrum of healthy to hip OA subjects. This allowed us to uncouple the association between the sagittal and axial plane kinematics in pre-THA patients. Patients exhibiting sagittal stiffness have been reported to be at risk of dislocation, due to increased flexion demands on the hip [25]. However, such patients may also be requiring extra rotation from the pelvis and hip for a given task, further increasing the impingement risk [26]. The range of rotation was more reduced when both hips were affected, whilst flexion did not show this trend. It is of interest that, although no differences were seen in spine and pelvic flexion between patients and controls, the axial kinematic data, particularly maximum ROM, was different. This is perhaps unsurprising, given that unilateral hip osteoarthritis and anterior osteophytosis would block the pelvis and femur at an earlier degree of flexion and would be independent of the flexion ability of the contralateral hip. In contrast, the rotation of the pelvis requires complementary movements of both hips to take place. Patients with bilateral disease were the ones illustrating the least rotation. This may be clinically relevant, because, in patients with bilateral disease that undergo unilateral THA, increased rotational stiffness of the pelvis is likely to remain, and that may place the THA at impingement risk. Further study is required to assess how these conditions change after THA and whether axial and sagittal kinematics remain different from that of controls. Additionally, studies on a larger number of cases grouped according to the preoperative characteristics before, but also after THA, will be welcome in the future to benefit from the dynamic motion analysis procedures detailed in the current study.

This study provides important information regarding the association between sagittal and axial kinematics and differences between patients with hip OA and healthy volunteers. The understanding of these interactions is of value to the arthroplasty surgeon, who has to take such kinematics into account when planning for a THA, in order to minimize complication and improve outcomes. Future THA-planning platforms may have to account for the axial plane kinematics in addition to the sagittal ones. However, this study also has some limitations. Firstly, the two groups were not matched for age. Despite our efforts, healthy volunteers were younger than OA pre-THA participants; we did not wish to reduce the power of the study by excluding the older patients for study inclusion to match for age. However, the age difference between groups was less than 10 years. This age difference has minimal effect on gait analysis and radiographic measurements [27]. Secondly, we applied strict inclusion criteria, especially for BMI inclusion. Reports have identified BMI as a factor influencing spinopelvic sagittal movement, with high BMI being associated with reduced flexion due to soft tissue impingement [1]. We were unable to test for such association as marker visibility can be significantly impeded in patients with large soft tissue envelope around their pelvis, precluding the ability of reliable data capture. Thirdly, only the lumbar and thoracic spine were studied in the motion analysis laboratory. The whole of the spine, including the cervical spine [28], has important interaction with regard to function, sagittal balance [29], and gaze during daily activities. Stiffness and abnormal and axial characteristics of the cervical spine are likely to be associated with certain thoracic 
and lumbar spine characteristics that would also influence kinematics. To date, there is little data to show cervical pathology influence on outcome following THA. Limited data have exhibited that patients with cervical arthrodesis have inferior outcome following THA, due to the presence of myelopathic features and abnormal spinopelvic characteristics [30]. However, it is evident that this is an area that further work is warranted.

\section{Conclusions}

In conclusion, this study illustrates that the motion analysis laboratory can perform reliable assessments of spinopelvic and hip characteristics. In doing so, the axial plane that is yet to be characterized can be included. The sagittal and axial pelvic range of movements correlates illustrating that pelvic rotation abnormalities are likely also contributing to the inferior outcomes seen in patients with abnormal spinopelvic flexion characteristics. Axial rotations of the pelvis and spine were least in patients with bilateral hip disease further emphasizing the importance of the hip-pelvic-spine interaction.

Author Contributions: Conceptualization, G.G.; methodology, D.S.C., M.L., and G.G.; software, D.S.C. and B.C.; formal analysis, D.S.C., M.L. and G.G.; investigation, D.S.C., M.L., and G.G.; data curation, D.S.C. and B.C.; writing —original draft preparation, D.S.C.; writing — review and editing, B.C., M.L. and G.G.; visualization, D.S.C., M.L. and G.G.; supervision, M.L.; project administration, M.L. and G.G.; funding acquisition, G.G. All authors have read and agreed to the published version of the manuscript.

Funding: This research received no external funding.

Institutional Review Board Statement: The study was conducted according to the guidelines of the Declaration of Helsinki, and approved by the Ottawa Health Science Network Research Ethics Board (20170312-01H, Apr-2019), and the University of Ottawa Research Ethic Board (H-05-19-4451, May-2019).

Informed Consent Statement: Informed consent was obtained from all subjects involved in the study.

Data Availability Statement: Data available on request due to privacy restrictions.

Acknowledgments: The authors wish to thank Erik Kowalski, Amy Murphy, Tejaswaree Beegodhur, Wantuir Ramos Junior and Flavia A. S. Mazuchi, from the Human Movement Biomechanics Laboratory (University of Ottawa, ON, Canada), for their support during data collection and data processing.

Conflicts of Interest: The authors declare no conflict of interest.

\section{Appendix A}

Table A1. Summary of kinematics ranges, and statistical significance between OA and Control groups, reporting mean (range).

\begin{tabular}{ccccc}
\hline Task & Parameters & OA (deg) & Control (deg) & $p$-Value \\
\hline \multirow{4}{*}{ SBR } & Max hip flexion & $78(46-111)$ & $99(83-119)$ & 0.012 \\
& Max spine flexion & $53(20-70)$ & $57(40-74)$ & 0.597 \\
& Sagittal spinal ROM & $36(12-57)$ & $39(31-53)$ & 0.782 \\
Max pelvic tilt & $5(-27-27)$ & $16(3-29)$ & 0.107 \\
Sagittal pelvic ROM & $30(7-54)$ & $39(30-54)$ & 0.113 \\
\hline \multirow{3}{*}{ STR } & Mean hip ROM & $3(-21-17)$ & $2(-15-28)$ & 0.067 \\
& Max spinal rotation affected side & $32(17-44)$ & $38(32-45)$ & $\mathbf{0 . 0 0 4}$ \\
& Max spinal rotation unaffected side & $32(18-47)$ & $38(31-43)$ & $\mathbf{0 . 0 2 0}$ \\
& Axial spinal ROM & $63(34-83)$ & $21(63-88)$ & $\mathbf{0 . 0 0 7}$ \\
& Max pelvic rotation affected side & $13(3-25)$ & $19(15-23)$ & $\mathbf{0 . 0 0 1}$ \\
& Max pelvic rotation unaffected side & $13(2-28)$ & $40(32-48)$ & $\mathbf{0 . 0 0 6}$ \\
\hline
\end{tabular}




\section{References}

1. Buckland, A.J.; Puvanesarajah, V.; Vigdorchik, J.; Schwarzkopf, R.; Jain, A.; Klineberg, E.O.; Hart, R.A.; Callaghan, J.J.; Hassanzadeh, H. Dislocation of a primary total hip arthroplasty is more common in patients with a lumbar spinal fusion. Bone Jt. J. 2017, 99, 585-591. [CrossRef] [PubMed]

2. Grammatopoulos, G.; Gofton, W.; Jibri, Z.; Coyle, M.; Dobransky, J.; Kreviazuk, C.; Kim, P.R.; Beaulé, P.E. 2018 Frank Stinchfield Award: Spinopelvic Hypermobility is Associated with an Inferior Outcome After THA: Examining the Effect of Spinal Arthrodesis. Clin. Orthop. Relat. Res. 2019, 477, 310-321. [CrossRef] [PubMed]

3. Innmann, M.M.; Merle, C.; Gotterbarm, T.; Ewerbeck, V.; Beaulé, P.E.; Grammatopoulos, G. Can spinopelvic mobility be predicted in patients awaiting total hip arthroplasty? A prospective, diagnostic study of patients with end-stage hip osteoarthritis. Bone Jt. J. 2019, 101, 902-909. [CrossRef] [PubMed]

4. Dorr, L.D.; Callaghan, J.J. Death of the Lewinnek "Safe Zone". J. Arthroplasty 2019, 34, 1-2. [CrossRef]

5. Feng, J.E.; Anoushiravani, A.A.; Eftekhary, N.; Wiznia, D.; Schwarzkopf, R.; Vigdorchik, J.M. Techniques for Optimizing Acetabular Component Positioning in Total Hip Arthroplasty. Defining a Patient-Specific Functional Safe Zone. JBJS Rev. 2019, 7, e5. [PubMed]

6. Elkins, J.M.; Callaghan, J.J.; Brown, T.D. The 2014 Frank Stinchfield Award: The "landing zone" for wear and stability in total hip arthroplasty is smaller than we thought: A computational analysis. Clin. Orthop. Relat. Res. 2014, 473, 441-452. [CrossRef] [PubMed]

7. Grammatopoulos, G.; Pandit, H.; Glyn-Jones, S.; McLardy-Smith, P.; Gundle, R.; Whitwell, D.; Gill, H.S.; Murray, D.W. Optimal acetabular orientation for hip resurfacing. J. Bone Jt. Surg.-Ser. B 2010, 92, 1072-1078. [CrossRef]

8. Grammatopoulos, G.; Thomas, G.E.R.; Pandit, H.; Beard, D.J.; Murray, D.W.; Gill, H.S. The effect of orientation of the acetabular component on outcome following total hip arthroplasty with small diameter hard-on-soft bearings. Bone Jt. J. 2015, 97, 164-172. [CrossRef]

9. Lewinnek, G.E.; Lewis, J.L.; Tarr, R.; Compere, C.L.; Zimmerman, J.R. Dislocations after total hip-replacement arthroplasties. J. Bone Jt. Surg.-Ser. A 1978, 60, 217-220. [CrossRef]

10. Grammatopoulos, G.; Mellon, S.J.; Chen, M.; Langton, D.; Pandit, H.; Murray, D.; Gill, H. The Effect of Pelvic Tilt on Acetabular Component Orientation in Hip Arthroplasty-A Radiological Analysis Using. In Proceedings of the ORS 2011 Annual Meeting, Los Angeles, CA, USA, 24-27 October 2011.

11. Yun, H.; Murphy, W.; Ward, D.; Zheng, G.; Hayden, B.; Murphy, S. Effect of pelvic tilt and rotation on cup orientation in standing anteroposterior radiographs. HIP Int. 2020, 30, 48-55. [CrossRef]

12. Abdel, M.P.; von Roth, P.; Jennings, M.T.; Hanssen, A.D.; Pagnano, M.W. What Safe Zone? The Vast Majority of Dislocated THAs Are Within the Lewinnek Safe Zone for Acetabular Component Position. Clin. Orthop. Relat. Res. 2016, 474, 386-391. [CrossRef]

13. Innmann, M.M.; Merle, C.; Phan, P.; Beaulé, P.E.; Grammatopoulos, G. How Can Patients with Mobile Hips and Stiff Lumbar Spines Be Identified Prior to Total Hip Arthroplasty? A Prospective, Diagnostic Cohort Study. J. Arthroplast. 2020, 35, S255-S261. [CrossRef] [PubMed]

14. Murray, D.W.; Fitzpatrick, R.; Rogers, K.; Pandit, H.; Beard, D.J.; Carr, A.J.; Dawson, J. The use of the Oxford hip and knee scores. J. Bone Jt. Surg.-Ser. B 2007, 89, 1010-1014. [CrossRef] [PubMed]

15. Nilsdotter, A.K.; Lohmander, L.S.; Klässbo, M.; Roos, E.M. Hip Disability and Osteoarthritis Outcome Score (HOOS)—Validity and Responsiveness in Total Hip Replacement. BMC Musculoskelet. Disord. 2003, 4, 1-8. [CrossRef] [PubMed]

16. Fairbank, J.C.T.; Pynsent, P.B. The oswestry disability index. Spine 2000, 25, 2940-2952. [CrossRef]

17. Ware, J.E.; Kosinski, M.; Keller, S.D. A 12-Item Short-Form Health Survey: Construction of Scales and Preliminary Tests of Reliability and Validity. Med. Care 1996, 34, 3. [CrossRef]

18. McDonnell, S.M.; Boyce, G.; Baré, J.; Young, D.; Shimmin, A.J. The incidence of noise generation arising from the large-diameter Delta Motion ceramic total hip bearing. J. Bone Jt. Surg.-Ser. B 2013, 95, 160-165. [CrossRef]

19. Pierrepont, J.W.; Feyen, H.; Miles, B.P.; Young, D.A.; Baré, J.V.; Shimmin, A.J. Functional orientation of the acetabular component in ceramic-on-ceramic total hip arthroplasty and its relevance to squeaking. Bone Jt. J. 2016, 98, 910-916. [CrossRef]

20. Mantovani, G.; Lamontagne, M. How different marker sets affect joint angles in inverse kinematics framework. J. Biomech. Eng. 2017, 139, 044503. [CrossRef]

21. Martin Bland, J.; Altman, D.G. Statistical methods for assessing agreement between two methods of clinical measurement. Lancet 1986, 327, 307-310. [CrossRef]

22. Zhou, X.; Wang, Q.; Zhang, X.; Chen, Y.; Shen, H.; Jiang, Y. Effect of Pelvic Obliquity on the Orientation of the Acetabular Component in Total Hip Arthroplasty. J. Arthroplast. 2012, 27, 299-304. [CrossRef] [PubMed]

23. Limmahakhun, S.; Box, H.N.; Arauz, P.; Hennessy, D.W.; Klemt, C.; Kwon, Y.M. In vivo analysis of spinopelvic kinematics and peak head-cup contact in total hip arthroplasty patients with lumbar degenerative disc disease. J. Orthop. Res. 2019, 37, 674-680. [CrossRef] [PubMed]

24. Fiorentino, N.M.; Atkins, P.R.; Kutschke, M.J.; Goebel, J.M.; Foreman, K.B.; Anderson, A.E. Soft tissue artifact causes significant errors in the calculation of joint angles and range of motion at the hip. Gait Posture 2017, 55, 184-190. [CrossRef]

25. Esposito, C.I.; Carroll, K.M.; Sculco, P.K.; Padgett, D.E.; Jerabek, S.A.; Mayman, D.J. Total Hip Arthroplasty Patients With Fixed Spinopelvic Alignment Are at Higher Risk of Hip Dislocation. J. Arthroplast. 2018, 33, 1449-1454. [CrossRef] 
26. Yun, H.; Murphy, W.S.; Ward, D.M.; Zheng, G.; Hayden, B.L.; Murphy, S.B. Effect of Pelvic Tilt and Rotation on Cup Orientation in Both Supine and Standing Positions. J. Arthroplast. 2018, 33, 1442-1448. [CrossRef]

27. Kowalski, E.; Catelli, D.S.; Lamontagne, M. Side does not matter in healthy young and older individuals-Examining the importance of how we match limbs during gait studies. Gait Posture 2019, 67, 133-136. [CrossRef]

28. Canseco, J.A.; Schroeder, G.D.; Patel, P.D.; Grasso, G.; Chang, M.; Kandziora, F.; Vialle, E.N.; Oner, F.C.; Schnake, K.J.; Dvorak, M.F.; et al. Regional and experiential differences in surgeon preference for the treatment of cervical facet injuries: A case study survey with the AO Spine Cervical Classification Validation Group. Eur. Spine J. 2021, 30, 517-523. [CrossRef] [PubMed]

29. Patwardhan, A.G.; Khayatzadeh, S.; Havey, R.M.; Voronov, L.I.; Smith, Z.A.; Kalmanson, O.; Ghanayem, A.J.; Sears, W. Cervical sagittal balance: A biomechanical perspective can help clinical practice. Eur. Spine J. 2018, 27, 25-38. [CrossRef] [PubMed]

30. Coyle, M.; Grammatopoulos, G.; Gofton, W.; Dobransky, J.; Kreviazuk, C.; Harris, N.; Lamontagne, M.; Phan, P.; Beaulé, P. The Presence of Cervical or Thoracic Arthrodesis is Associated with Inferior Outcome Post-THA. In Proceedings of the ORS Annual Meeting, Austin, TX, USA, 2-5 February 2019. 